\title{
滴涂法制备石墨烯/碳纳米管复合薄膜及其表征
}

\author{
黄 维, 朱家艺, 李 浩, 杨䂀, 王朝阳, 付志兵 \\ (中国工程物理研究院激光聚变研究中心, 绵阳 621900)
}

摘 要: 以化学气相沉积(CVD)法制备的石墨烯和碳纳米管的邻二氯苯分散液为原料, 采用滴涂法制备石墨烯/碳纳 米管复合薄膜, 用扫描电子显微镜(SEM)、透射电子显微镜(TEM)、拉曼光谱(Raman)和 X 射线光电子能谱(XPS) 对其形貌和结构进行表征。实验发现随着碳纳米管分散液浓度的增大, 复合薄膜结构中碳纳米管的面密度线性增 大。利用紫外-可见光谱仪和四探针测试仪表征了不同碳纳米管浓度下复合薄膜的透光率及其薄层电阻，结果表明： 随着碳纳米管浓度的增大, 复合薄膜的透光率及其薄层电阻都将减小, 当碳纳米管浓度为 $0.1 \mathrm{mg} / \mathrm{mL}$ 时, 复合薄膜 的透光率 $(550 \mathrm{~nm}$ ) 及其薄层电阻分别为 $92.18 \%$ 和 $0.998 \mathrm{k} \Omega /$ 。。实验通过调节碳纳米管浓度制备得到不同性能的石 墨烯/碳纳米管复合薄膜，该复合薄膜在透明电极、场效应晶体管和激光锁模等方面具有潜在应用。

关 键 词: 石墨烯; 碳纳米管; 复合薄膜; 滴涂法

中图分类号: TB332 文献标识码: A

\section{Preparation and Characterization of Graphene/Carbon Nanotube Hybrid Thin Films by Drop-coating}

\author{
HUANG Wei, ZHU Jia-Yi, LI Hao, YANG Xi, WANG Chao-Yang, FU Zhi-Bing \\ (Research Center of Laser Fusion, China Academy of Engineering Physics, Mianyang 621900, China)
}

\begin{abstract}
Hybrid thin films were prepared via a drop-coating method by using graphene prepared by chemical vapor deposition(CVD) and carbon nanotube dispersed in 1, 2-dichlorobenzene as crude materials. The structure and morphology of hybrid thin films were characterized by scanning electron microscope (SEM), transmission electron microscopy (TEM), Raman spectroscopy and X-ray photoelectron spectroscopy (XPS). It was found that surface density of carbon nanotube in the hybrid thin films increased linearly with the increase of dispersion concentration. Furthermore, the transmittance and sheet resistance of the hybrid thin films, which were characterized by UV-visible spectroscope and four-point probe analyzer, respectively, both significant decreased when concentration of the dispersed carbon nanotube increased. The two parameters, to be specific, were found to be $92.18 \%$ (at $550 \mathrm{~nm}$ ) and 0.998 $\mathrm{k} \Omega / \square$, respectively as the concentration of the dispersed carbon nanotube reaching $0.1 \mathrm{mg} / \mathrm{mL}$. In other words, the performances of the hybrid thin films can be improved by regulating concentration of carbon nanotube in the dispersion, showing the films potential applications in the fields of transparent electrodes, field effect transistors and laser mode-locking.
\end{abstract}

Key words: graphene; carbon nanotube; hybrid thin films; drop-coating

收稿日期: 2016-05-10; 收到修改稿日期：2016-08-15

基金项目: 中国工程物理研究院科学技术发展基金(2015B0302003); 国家自然科学基金青年科学基金(51502274); 中国博 士后科学基金(2015M572499)

Science and technology Development fund of China Academy of Engineering Physics (2015B0302003); National Natural Science Funds of China (51502274); Postdoctoral Science Foundation of China (2015M572499)

作者简介: 黄 维(1990-), 男, 硕士研究生. E-mail: huangbiqian1990319@126.com

通讯作者: 王朝阳, 研究员. E-mail: wangchy807@caep.cn; 付志兵, 副研究员. E-mail: fuzhibingcn@163.com 
碳纳米管和石墨烯是典型的一维和二维碳纳米 材料, 自 1991 年 ${ }^{[1]}$ 和 2004 年 ${ }^{[2]}$ 首次报道以来引起了 广泛关注。石墨烯和碳纳米管具有优异的机械性能、

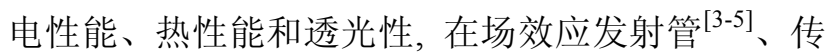
感器 ${ }^{[6-7]}$ 、电子发射 ${ }^{[8]}$ 和激光锁模 ${ }^{[9-11]}$ 等方面有潜在 的应用。

化学气相沉积(CVD)法可在特定基底(如铜和 镍)上制备出高质量、大面积和连续的石墨烯薄膜, 获得的石墨烯薄膜结构完整、质量良好, 并可以通 过控制工艺参数来调节其在基底上的生长厚度, 将 其转移至目标基底上实现其应用 ${ }^{[12-14]}$ 。但是 CVD 法制备的石墨烯会存在大量的晶界缺陷, 导致石墨 烯的导电性下降, 且转移过程易碎裂。石墨烯薄膜 与碳纳米管复合可以得到纯碳复合薄膜, 在保证薄 膜透光性的同时, 碳纳米管将弥补石墨烯的晶界缺 陷，从而提高薄膜的机械性能和导电性。Song 等 ${ }^{[15]}$ 通过在铜䈃上旋涂碳纳米管再生长石墨烯得到纯碳 复合薄膜, 该复合薄膜的导电性得到很大提高, 透 光性为 $96.4 \%$ 时薄层电阻仅为 $300 \Omega /$ 。滴定涂膜法 具有成本低、操作简单等优点, 且碳纳米管邻二氯 苯分散液是粘度系数很小的悬浊液, 相比旋转涂膜 法和提拉成膜法得到的复合薄膜, 滴定涂膜法分散 更加均匀可控 ${ }^{[16]}$ 。通过控制分散液浓度得到不同碳 纳米管面密度、透光度、导电性的复合薄膜, 该复 合薄膜在透明电极、场效应晶体管 ${ }^{[15]}$ 和激光锁模 ${ }^{[17]}$ 等方面具有很大的应用前景。本工作采用常压 CVD 法, 以铜䈃为基底、乙醇为碳源生长石墨烯薄膜, 将 碳纳米管邻二氯苯分散液滴定涂膜在石墨烯上得到 复合薄膜。

\section{1 实验方法}

\section{1 石墨烯薄膜的制备}

铜䇴(厚度: $25 \mu \mathrm{m}$,纯度: $99.8 \%$, No. 13382, 阿法 埃莎中国化学有限公司)用辊压机压平整, 浸入硝 酸溶液中 $(5.4 \%(w / w))$ 预处理 $30 \mathrm{~s}$, 以除去铜箔上的 杂质颗粒 ${ }^{[18]}$ 。处理过的铜䇴置于石英载片上作为石 墨烯的生长基底。抽真空至约 $5 \mathrm{~Pa}$ 后通入第 1 路 $\mathrm{Ar} / \mathrm{H}_{2}\left(5 \%\right.$ 的 $\left.\mathrm{H}_{2}\right)$ 混合气至常压, 重复上述过程以降 低氧含量, 防止高温条件下铜䈃被氧化。保持 $\mathrm{Ar} / \mathrm{H}_{2}$ 混合气 $550 \mathrm{sccm}$ 流量不变, 开启程序控制 $60 \mathrm{~min}$, 常压下石英管恒速升温至 $1000^{\circ} \mathrm{C}$, 随后保温预热处 理 $0.5 \mathrm{~h}$ 。预热结束后, 用第 2 路 $\operatorname{Ar}(80 \mathrm{sccm})$ 气将乙 醇(纯度: $99 \%$, 上海阿拉丁生化科技股份有限公司) 作为碳源带入石英管内, 保持一段时间用于乙醇的
裂解沉积, 随即快速冷却至室温得到石墨烯薄膜沉 积于铜䈃上。

\section{2 石墨烯/碳纳米管复合薄膜的制备}

CVD 法在铜箔上生长石墨烯薄膜，铜箔背面也 会沉积不完整的石墨烯，首先用橡皮擦和酒精除去 背面的石墨烯薄膜 ${ }^{[19]}$ 。配置溶度分别为 $0.02 、 0.04$ 、 0.06、0.08、0.1 mg/mL 的碳纳米管(长度: $5 \sim 30 \mu \mathrm{m}$, 直径: 1 2 nm, 纯度: $>95 \%$, No.XFS22, 南京先丰纳 米材料科技有限公司)邻二氯苯分散液，取分散液 $0.01 \mathrm{~mL}$ 滴涂在石墨烯/铜箔上，分散液完全铺展， 在室温下自然晾干。为了保证复合薄膜的纯度, 复 合薄膜转移过程中不旋涂任何保护层 ${ }^{[20]}$, 具体操作 为: 配置 $0.1 \mathrm{~mol} / \mathrm{L}$ 的 $\mathrm{FeCl}_{3}$ 溶液作为铜䈃刻蚀液, 将干燥后的碳纳米管/石墨烯/铜䈃漂浮(经过处理的 干净一面朝下)在刻蚀液中。反应在加热板上(温度 约为 $50^{\circ} \mathrm{C}$ )进行, 经过大约 $4 \mathrm{~h}$ 铜䈃被完全刻蚀, 得 到的复合薄膜漂浮在刻蚀液上。用表面血. (直径 $40 \mathrm{~mm}$ )将漂浮的复合薄膜捞至去离子水中多次清 洗，除去残留的刻蚀液，最后将清洗干净的复合薄 膜转移至 $\mathrm{K} 9$ 玻璃或 $\mathrm{SiO}_{2}(300 \mathrm{~nm}) / \mathrm{Si}(100)$ 晶片基底 上进行相应的表征。

\section{3 样品表征}

采用扫描电子显微镜(SEM) JSM-7000F (日本电 子光学公司)和透射电子显微镜(TEM)Fecnai F30 (荷兰 Philips-FEI 公司) 观察碳基薄膜的表面形貌; 用拉曼光谱仪(Raman) HR800(法国 Horiba Jobin Yvon LabRam 公司)进行薄膜的结构分析; 紫外可 见光谱仪(UV/Vis)Carry 5000(美国 VARIAN 公司) 用于测量薄膜的透光性; 采用 $\mathrm{X}$ 射线光电子能谱仪 (XPS)K-alpha(美国 Thermo Fisher Scientific 公司) 进行薄膜的元素分析; 采用接触角测量仪 OCA15EC(德国 dataphysics 公司)测定分散液与薄膜 的接触角; 四角探针测试仪 RTS- 8 (天津综科科技 有限公司)测定薄膜的薄层电阻。

\section{2 结果与讨论}

\section{1 石墨烯的 SEM 表征}

图 1 是退火铜䇴和石墨烯/铜箔的 SEM 照片和 $\mathrm{EDX}$ 图谱。图 1(a)为 $\mathrm{Ar} / \mathrm{H}_{2}$ 混合气退火 $0.5 \mathrm{~h}$ 处理 后铜箔的表面形貌，其中的白色亮斑为杂质颗粒， EDX 分析显示, 白色亮斑处只含有铜元素和氧元素, 推断杂质颗粒可能是铜的氧化物或铜表面缺陷。图 1(b)为经过硝酸预处理再退火的铜箔表面形貌，铜 箔表面干净, 白色亮斑消失, 可能原因是稀硝 

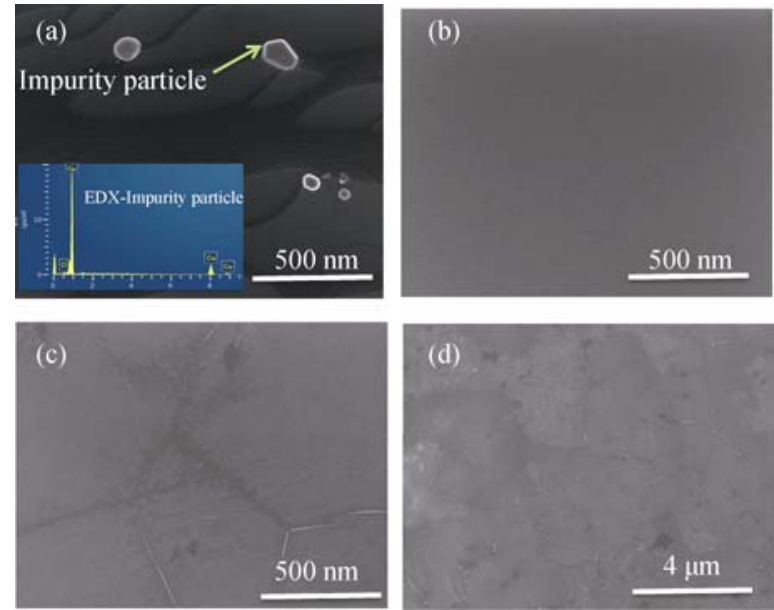

图 1 未处理(a)和硝酸预处理(b)退火后的铜䈃的 SEM 照片, 石墨烯/铜䇴的高倍(c)和低倍(d)SEM 照片

Fig. 1 SEM images of the pristine copper foil (a) and nitric acid pre-cleaned copper foil (b) after annealing, high- (c) and low- (d) magnification SEM images of graphene $/ \mathrm{Cu}$

酸与铜䇴表面反应产生的 $\mathrm{NO}_{2}$ 气体除去杂质颗粒, 使铜管表面平整 ${ }^{[18]}$ 。图 1(c)、(d)是石墨烯/铜䈃不同 放大倍率的 SEM 照片, 其中白色线条为石墨烯的 褶皱, 黑色线条为铜箔晶界 ${ }^{[21]}$, 可以看出沉积的石 墨烯薄膜结构完整, 连续性、均一性较好。

\section{2 碳纳米管分散体系的选择}

碳纳米管具有纳米粉体的强团聚效应和纤维 材料的纠缠粘结现象, 各种效应的叠加使得碳纳
米管分散特别困难。可以选择具有离域 $\pi$ 键的溶剂 体系通过 $\pi-\pi$ 非共价作用稳定碳纳米管分散状态, 通过超声处理破坏长纤维纠缠粘结状态, 克服团 聚体强吸附力, 从而得到均匀的碳纳米管分散液 ${ }^{[22]}$ 。

图 2(a)是不同碳纳米管分散体系超声处理 $8 \mathrm{~h}$ 后静置 一段时间后的情形, 碳纳米管浓度均为 $0.1 \mathrm{mg} / \mathrm{mL}$ 。 从图 2(a)中可以看出二甲基甲酰胺(DMF)、N-甲基 吡咯烷酮(NMP)分散液中的碳纳米管出现了严重 的聚沉现象, 十二烷基苯磺酸钠(SDBS)水溶液和 邻二氯苯分散液中的碳纳米管能够均匀稳定存在, 这是由于 SDBS 表面活性剂和邻二氯苯含有苯环 结构, 具有更强的离域 $\pi$ 键，与碳纳米管之间作 用力更强。图 2(b)是碳纳米管邻二氯苯分散液滴 在导电胶上干燥后的 SEM 照片, 从图中可以看出 碳纳米管单根分散效果很好, 没有出现聚集。图 2(c) 是石墨烯薄膜与不同分散液体系接触角测量 (测量方法为悬滴法, 接触角分析方法为 $\theta / 2$ 法)的 照片, 可以看出邻二氯苯与石墨烯有很强的亲和 力, 滴在石墨烯薄膜上能够完全铺展。为了得到石 墨烯/碳纳米管纯碳复合薄膜, 应选择有机溶剂作 为分散剂, 并且邻二氯苯对石墨烯薄膜的润湿性 最优，综上考虑邻二氯苯作为碳纳米管的分散剂 效果最佳。

\section{3 石墨烯/碳纳米管复合薄膜的结构表征}

图 3(a e) 为不同碳纳米管浓度复合薄膜的 SEM
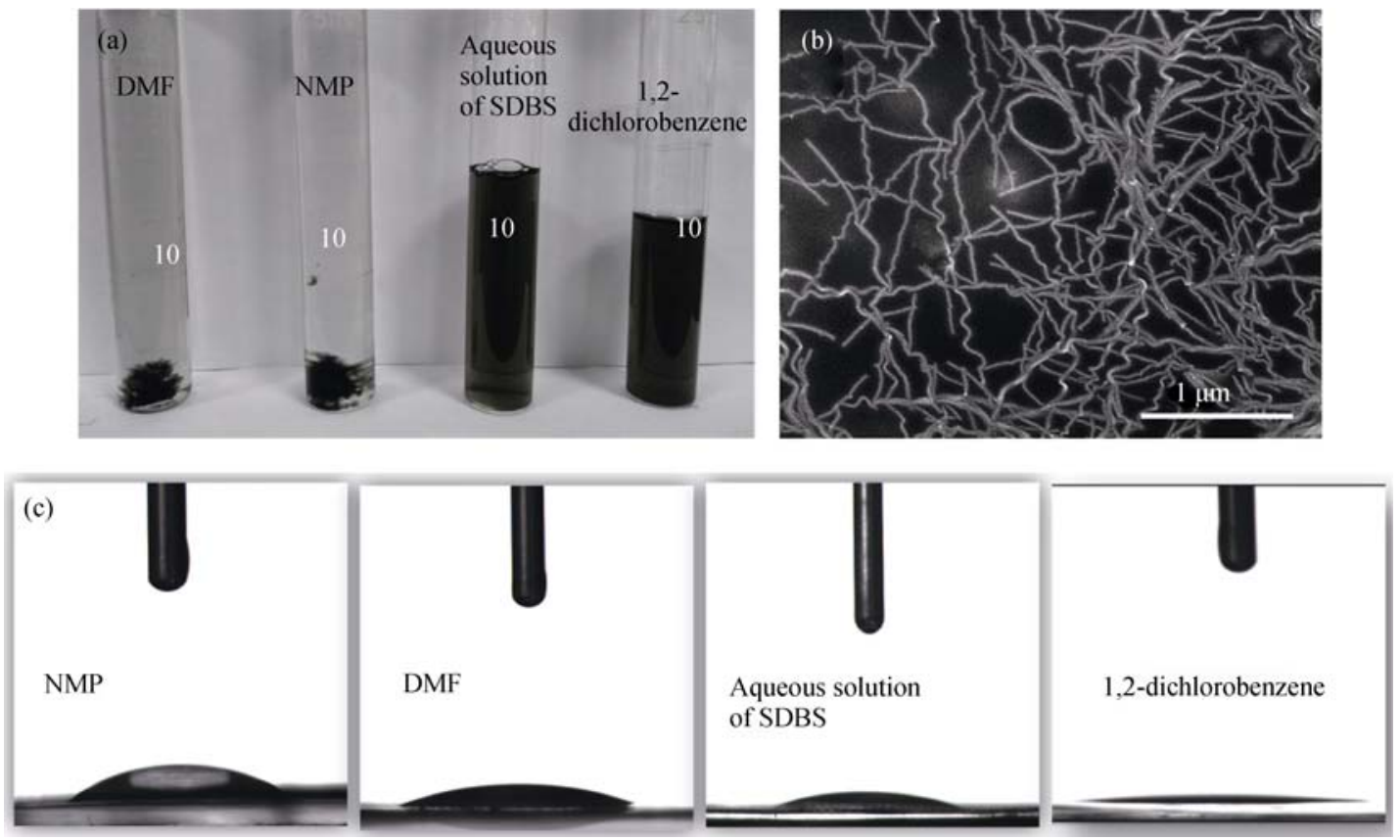

图 2 (a)不同碳纳米管分散体系的照片, (b)分散的碳纳米管 SEM 照片和(c)石墨烯薄膜与不同分散液接触角测量的照片

Fig. 2 (a) Photo of carbon nanotube dispersed in different dispersion system, (b) SEM image of dispersive carbon nanotube, and (c) schematic pictures of contact angle measurement of graphene films with different dispersion 

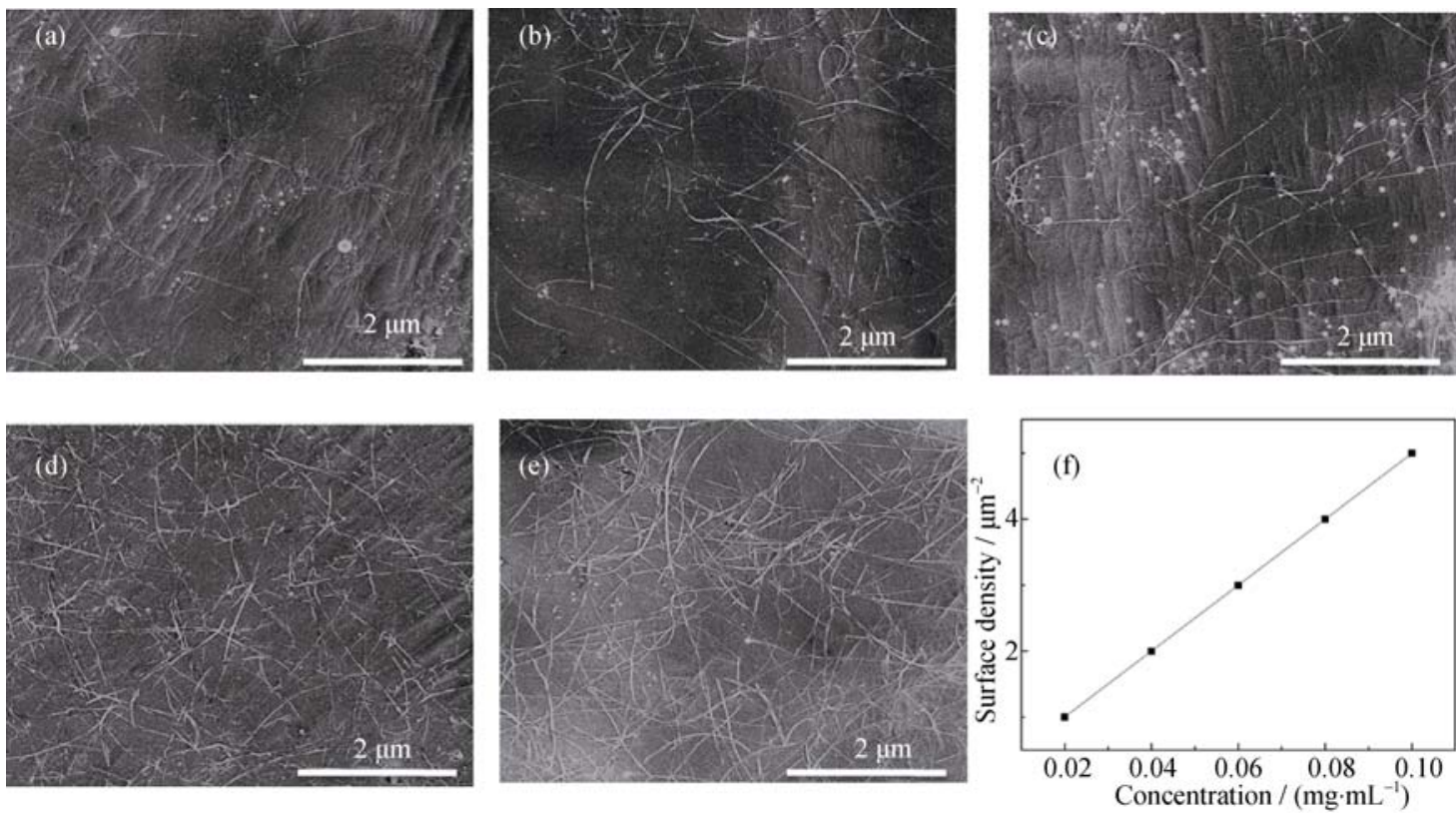

图 3 不同碳纳米管浓度下复合薄膜的 SEM 照片(a e), 碳纳米管面密度随分散液浓度的变化曲线(f)

Fig. 3 SEM images of hybrid thin films with different concentration of carbon nanotube (a-e), and curve of surface density of carbon nanotube $v s$ concentration changes of dispersion (f)

Carbon nanotube concentration (a-e): 0.02, 0.04, 0.06, 0.08, $0.1 \mathrm{mg} / \mathrm{mL}$

照片, 从图中可以看出碳纳米管在石墨烯薄膜上分 散均匀, 分散液浓度低时碳纳米管呈单根分散, 随 着分散液浓度的增大, 碳纳米管的交联性增加, 碳 纳米管的面密度分别约为 $1 、 2 、 3 、 4 、 5$ 根/平方微 米。如图 3(f)所示, 碳纳米管的面密度随分散液浓度 的增加呈线性增大。

图 4(a d)为石墨烯/碳纳米管复合薄膜(浓度为 $0.1 \mathrm{mg} / \mathrm{mL}$ ) 的 TEM 照片。当利用高分辨 TEM 观察 石墨烯薄膜层片边缘时, 可直接观察到石墨烯薄膜 的层数, 由图 4(a)所示区域可见石墨烯薄膜大约为 2 3 层。图 4(b)为图 4(a)中薄膜边缘的电子衍射谱, 显示出石墨烯中碳原子呈六边形排列, 可观察到六 边形每个顶点有两个衍射点, 表明石墨烯为双层。

图 4(c)为复合薄膜的低倍 TEM 照片, 从图中可以看 出石墨烯完整性很好, 褶皱清晰可见, 石墨烯薄膜 有部分位置折叠(转移过程中造成), 碳纳米管在石 墨烯薄膜上有少量交错分布, 且有少量聚集成碳纳 米管束。图 4(d) 为碳纳米管的高倍的 TEM 照片, 从 图中可以估计碳纳米管的管壁层数为 4 层左右, 厚 度约为 $5 \mathrm{~nm}$ 。

图 5 为在去离子水中清洗后的复合薄膜转移至 $\mathrm{SiO}_{2}(300 \mathrm{~nm}) / \mathrm{Si}(100)$ 晶片基底上进行 XPS 表征得到 的结果, 从图谱中可以看出复合薄膜清洗得干净, 没有刻蚀液中的金属离子杂质。

采用 Raman 光谱(激发波长 $514 \mathrm{~nm}$ )表征石墨 烯、石墨烯/碳纳米管复合薄膜的层数和质量, 如图
6 所示。石墨烯和碳纳米管具有典型的特征峰：D 峰、 $\mathrm{G}$ 峰和 $2 \mathrm{D}$ 峰 ${ }^{[23]}$, 当共振拉曼谱的激发波长为 $514 \mathrm{~nm}$ 时, 碳纳米管在 $180 \mathrm{~cm}^{-1}$ 附近存在 RBM 峰, 而石墨烯不存在 ${ }^{[24]}$ 。图 6 中位于 $1350 \mathrm{~cm}^{-1}$ 附近的 D 峰反映了石墨烯片层的无序性; 位于 $1580 \mathrm{~cm}^{-1}$ 附 近的 $\mathrm{G}$ 峰是碳 $\mathrm{SP}^{2}$ 结构的特征峰, 反映其对称性和 结晶程度; 而 $2700 \mathrm{~cm}^{-1}$ 附近的 2D 峰源于两个双声
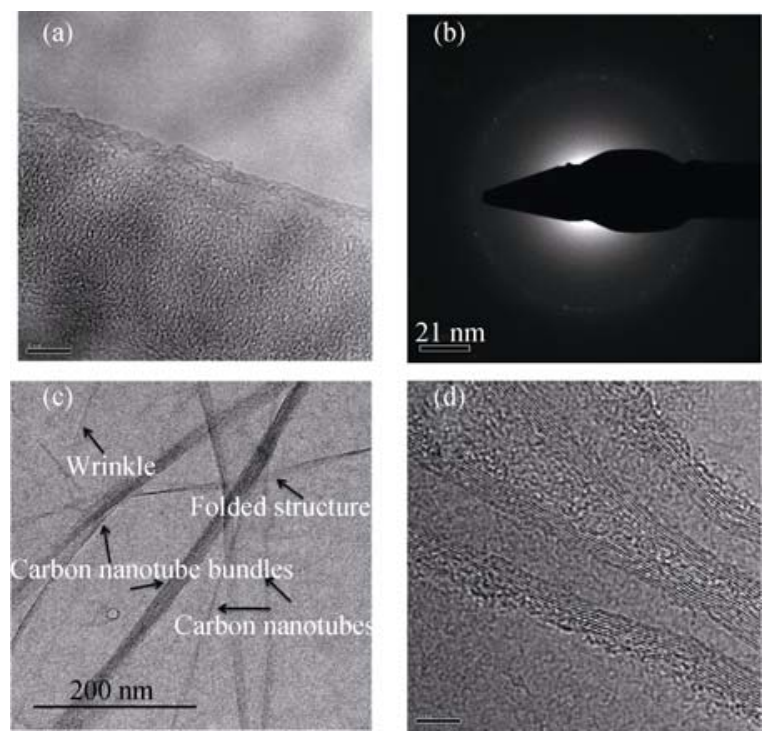

图 4 石墨烯的高倍 TEM 照片(a)和电子衍射谱(b), 石墨烯/ 碳纳米管复合薄膜的低倍(c)和高倍(d)TEM 照片

Fig. 4 High-magnification TEM image (a) and electron diffraction pattern (b) of graphene, and low-magnification (c) and high-magnification (d) TEM images of hybrid thin films 


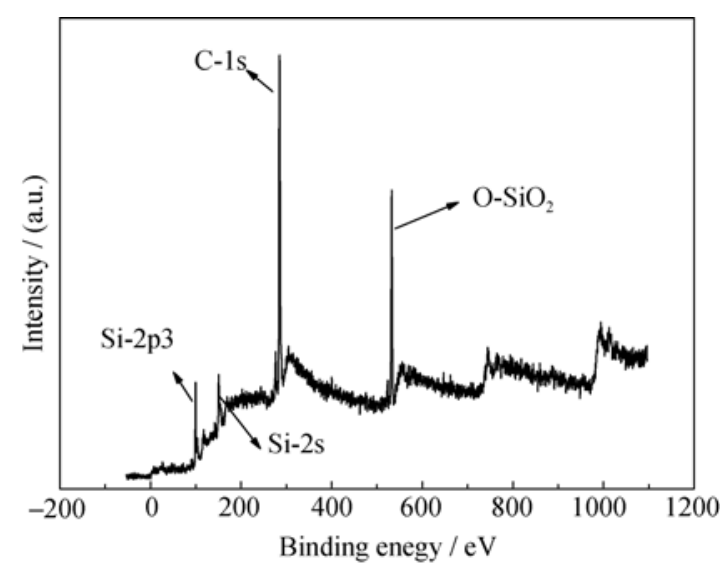

图 5 碳纳米管/石墨烯/ $\mathrm{SiO}_{2}$ 的 XPS 图谱

Fig. 5 XPS spectrum of carbon nanotube/graphene/ $/ \mathrm{SiO}_{2}$

子非弹性散射 ${ }^{[25]}$ 。此外, 石墨烯的层数可通过 2D 峰与 $\mathrm{G}$ 峰的强度比值和 $2 \mathrm{D}$ 峰的半高宽来推断 ${ }^{[26]}$ 。

图 6(c)中 D 峰的强度很低, 说明石墨烯的缺陷很少, 薄膜连续且完整; $I_{\mathrm{G}} / I_{2 \mathrm{D}}$ 为 0.64 , 且 $2 \mathrm{D}$ 峰的半高宽为 $52.3 \mathrm{~cm}^{-1}$, 可推断石墨烯为双层。图 6(b)中 $180 \mathrm{~cm}^{-1}$ 附近的 RBM 峰为碳纳米管的特征峰, 证明实验获得 了碳纳米管/石墨烯复合薄膜，图 6(a)和 6(b) $140 \mathrm{~cm}^{-1}$ 附近的峰源自仪器本身的干扰峰。
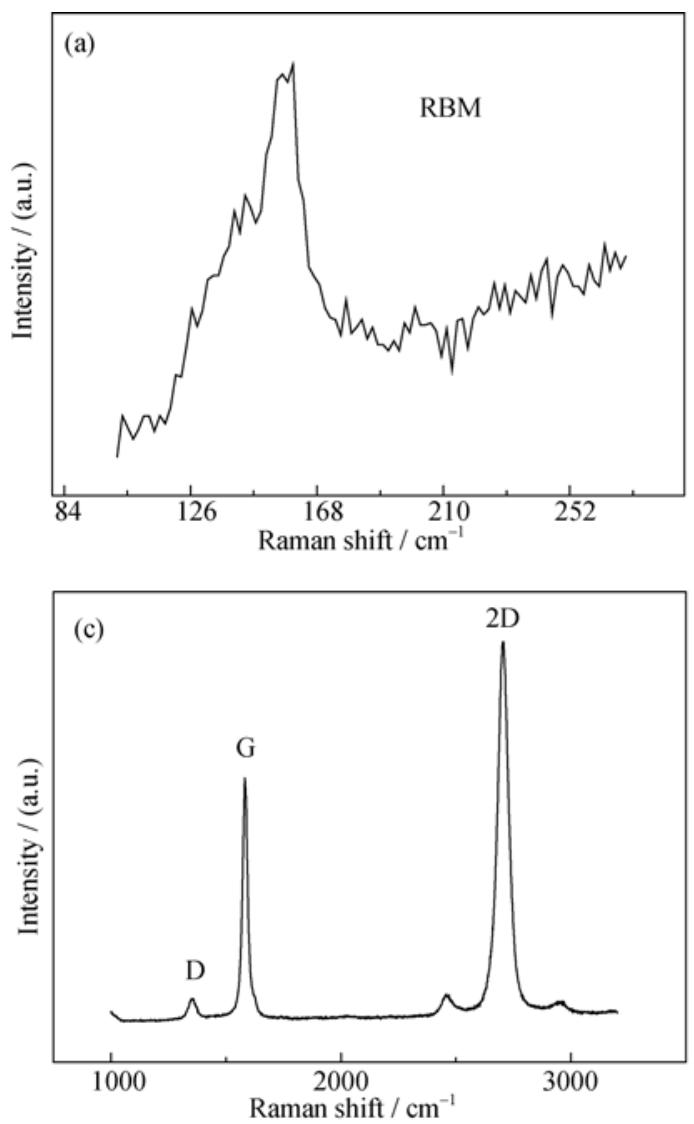

\section{4 复合薄膜的透光性与薄层电阻测量}

将制备的复合薄膜直接湿法转移至 $\mathrm{K} 9$ 玻璃上 测定其透光性和薄层电阻, 结果如图 7(a)所示。本 文实验条件下制备的石墨烯透光率为 $95.93 \%$ (在 $550 \mathrm{~nm}$ 处), 薄层电阻为 $2.374 \mathrm{k} \Omega /$ 口, 由石墨烯在可 见光区的不透光度为 $(2.3 \pm 0.1 \%) \times \mathrm{N}^{[27]}(\mathrm{N}$ 为石墨烯 层数)可以推断得到的石墨烯为双层。采用滴涂法制 备不同碳纳米管浓度的复合薄膜的透光性曲线如图 7(b)所示，由图可知复合薄膜在可见光区均具有很 好的透光性。当碳纳米管浓度分别为 $0.02 、 0.04$ 、 0.06、0.08、0.1 mg/mL 时，复合薄膜的透光性(在 $550 \mathrm{~nm}$ 处)分别为 $95.08 \% 、 93.89 \% 、 93.21 \% 、 92.56 \%$, $92.18 \%$, 薄层电阻分别为 $1.946 、 1.490 、 1.232 、 1.174$ 、 $0.998 \mathrm{k} \Omega /$ 口。复合薄膜的透光性和薄层电阻对碳纳 米管浓度作图，得到图 7(c), 由图可知随着滴涂碳 纳米管浓度的增加，薄膜的透光性逐渐减小，这是 由于碳纳米管浓度的增加，增大了复合薄膜中碳纳 米管的面密度，而碳纳米管在可见光区具有一定的 不透光性，因此造成薄膜的透光性下降。由图 7(c) 还可知，随着碳纳米管浓度的增大，复合薄膜的薄 层电阻逐渐减小，这是因为碳纳米管面密度的增大
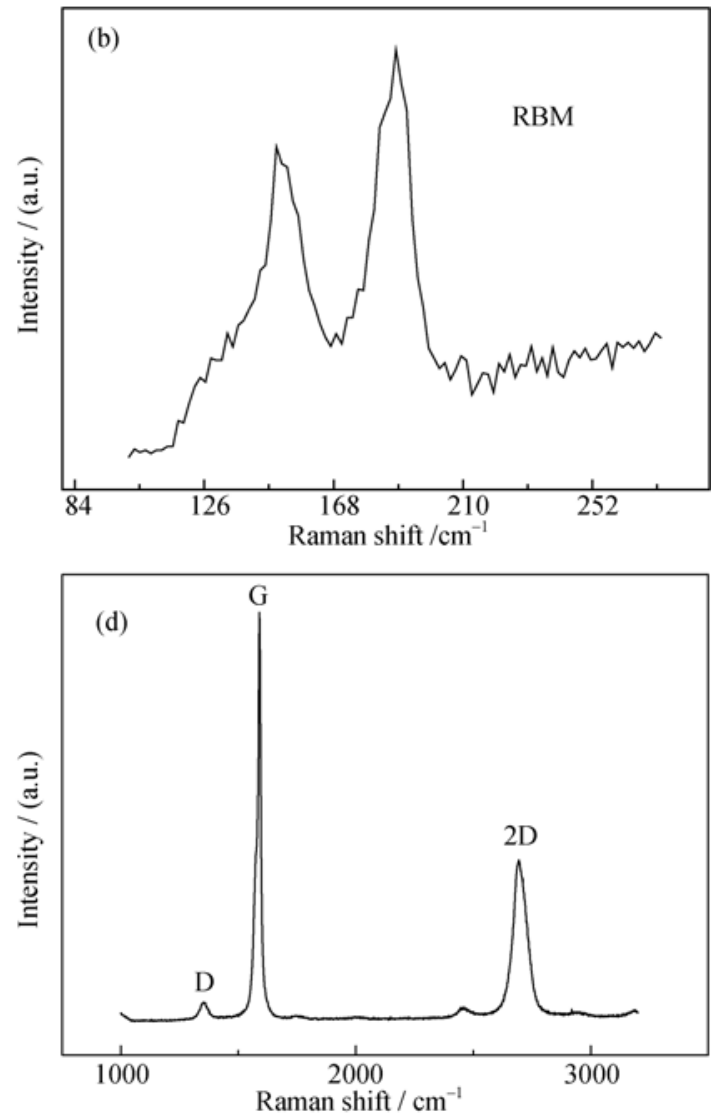

图 6 石墨烯 $(a, c)$ 和石墨烯/碳纳米管复合薄膜 $(b, d)$ 的拉曼光谱图

Fig. 6 Raman spectra of graphene films (a,c) and graphene/carbon nanotube hybrid films (b,d) (a and b) RBM of graphene and graphene/ carbon nanotube hybrid films, respectively 

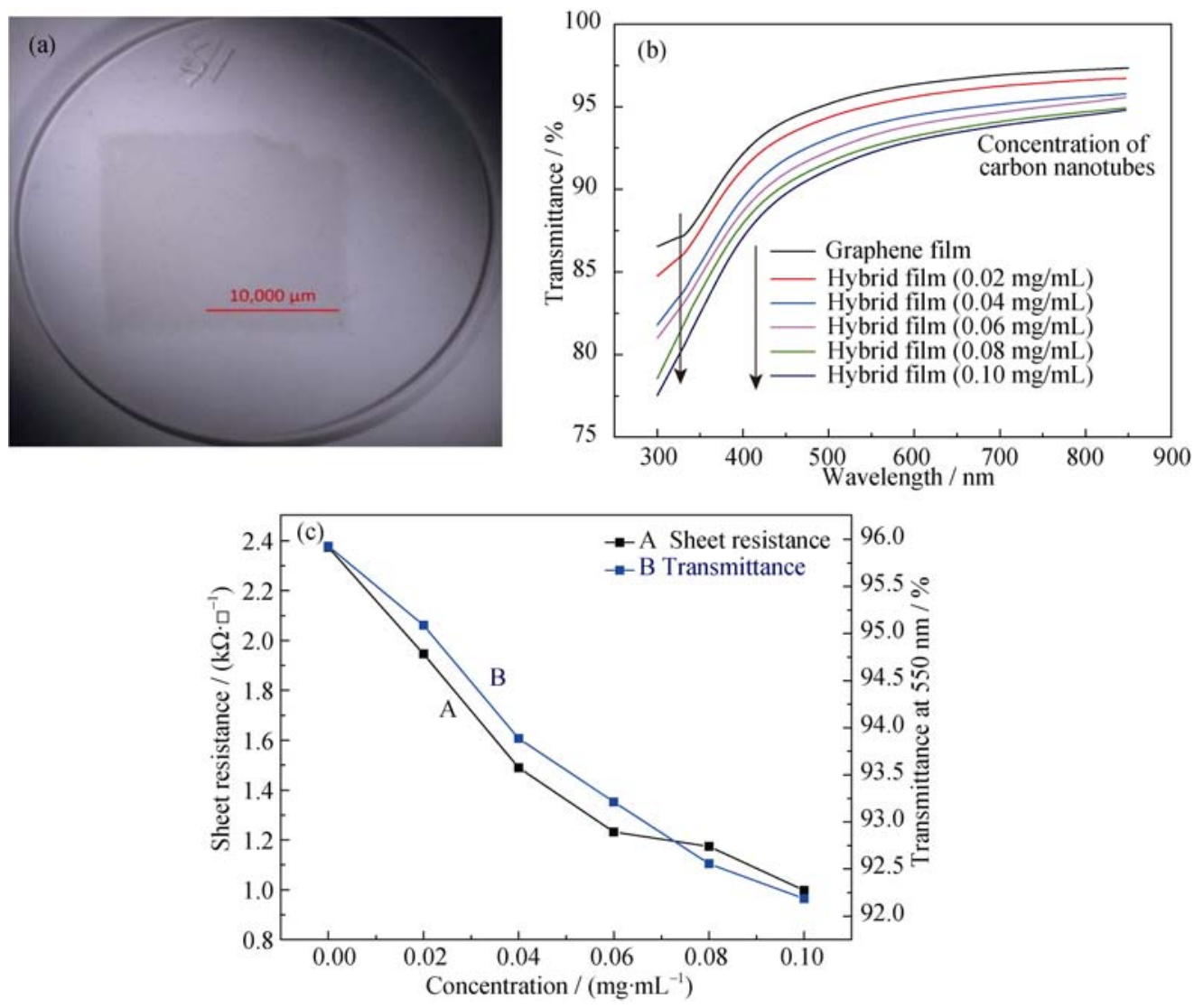

图 7 转移至 $\mathrm{K} 9$ 玻璃上的复合薄膜的照片(a), 不同碳纳米管浓度下复合薄膜的透光性曲线(b)及复合薄膜的透光率和薄层电阻 随碳纳米管浓度的变化曲线(c)

Fig. 7 Image of hybrid film transferred on K9 glass (a), transmittance curve of hybrid thin films with different concentrations of carbon nanotube (b), and curve of transmittance and sheet resistance of hybrid films $v s$ concentration changes of carbon nanotube (c)

促进了石墨烯晶界的电子传输和消除晶界处的缺陷 散射 ${ }^{[15]}$, 从而导致复合薄膜的薄层电阻减小。

\section{3 结论}

1) 采用经过硝酸预处理的铜䇴为基底，通过 常压 CVD 法获得了大面积、结构完整且质量良好 的石墨烯薄膜, 该薄膜的透光率 $(550 \mathrm{~nm})$ 及薄层电 阻分别为 $95.93 \%$ (在 $550 \mathrm{~nm}$ 处)和 $2.374 \mathrm{k} \Omega / \square$ 。

2) 通过比较 DMF、NMP、SDBS 水溶液和邻 二氯苯等多种溶剂, 发现邻二氯苯对碳纳米管的分 散及石墨烯薄膜的润湿效果最佳。

3) 采用 Raman、SEM 和 TEM 等技术表征了复 合薄膜的结构, 结果表明碳纳米管均匀分布在石墨 烯薄膜上, 且随着碳纳米管浓度的增大, 其在石墨 烯薄膜上的面密度线性增大。

4) 通过调节碳纳米管的浓度, 可以调控复合 薄膜的透光性和导电性。随着碳纳米管浓度的增加, 复合薄膜的透光性减小、导电性增加。

\section{参考文献:}

[1] LIJIMA S. Helical microtubules of graphitic carbon. Nature, 1991, 354(6348): $56-58$

[2] NOVOSELOV K S, GEIM A K, MOROZOV S V, et al. Electric field effect in atomically thin carbon films. Science, 2004, 306(5696): 666-669.

[3] SCHWIERZ F. Graphene transistors. Nature Nanotechnology, 2010, 5(7): 487-496.

[4] GOMEZ-NAVARRO C, WEITZ R T, BITTNER A M, et al. Electronic transport properties of individual chemically reduced graphene oxide sheets. Nano Letters, 2007, 7(11): 3499-3503.

[5] TUNG V C, ALLEN M J, YANG Y, et al. High-throughput solution processing of large-scale graphene. Nature Nanotechnology, 2009, 4(1): 25-29.

[6] ANG P K, CHEN W, WEE A T S, et al. Solution-gated epitaxial graphene as pH sensor. Journal of the American Chemical Society, 2008, 130(44): 14392-14393.

[7] DUA V, SURWADE S P, AMMU S, et al. All - organic vapor sensor using inkjet - printed reduced graphene oxide. Angewandte 
Chemie International Edition, 2010, 49(12): 2154-2157.

[8] SOIN N, SINHA ROY S, Roy S, et al. Enhanced and stable field emission from in situ nitrogen-doped few-layered graphene nanoflakes. The Journal of Physical Chemistry C, 2011, 115(13): 5366-5372.

[9] SUN Z, HASAN T, TORRISI F, et al. Graphene mode-locked ultrafast laser. ACS Nano, 2010, 4(2): 803-810.

[10] HUANG P L, LIN S C, YEH C Y, et al. Stable mode-locked fiber laser based on CVD fabricated graphene saturable absorber. Optics Express, 2012, 20(3): 2460-2465.

[11] WANG F, ROZHIN A G, SCARDCCI V, et al. Wideband-tuneable, nanotube mode-locked, fibre laser. Nature Nanotechnology, 2008, 3(12): 738-742.

[12] REINA A, JIA X, HO J, et al. Large area, few-layer graphene films on arbitrary substrates by chemical vapor deposition. Nano Letters, 2008, 9(1): 30-35.

[13] KIM K S, ZHAO Y, JANG H, et al. Large-scale pattern growth of graphene films for stretchable transparent electrodes. Nature, 2009, 457(7230): 706-710.

[14] LI X, CAI W, AN J, et al. Large-area synthesis of high-quality and uniform graphene films on copper foils. Science, 2009, 324(5932): $1312-1314$

[15] KIM S H, SONG W, JUNG M W, et al. Carbon nanotube and graphene hybrid thin film for transparent electrodes and field effect transistors. Advanced Materials, 2014, 26(25): 4247-4252.

[16] TAO Z, WANG B, HOU G, et al. Preparation of $\mathrm{BaZr}_{0.1} \mathrm{Ce}_{0.7} \mathrm{Y}_{0.2} \mathrm{O}_{3-\delta}$ thin embrane based on a novel method-drop coating. International Journal of Hydrogen Energy, 2014, 39(28): 16020-16024.

[17] BAO Q, ZHANG H, YANG J, et al. Graphene-polymer nanofiber membrane for ultrafast photonics. Advanced Functional Materials, 2010, 20(5): 782-791.
[18] KIM S M, HSU A, LEE Y H, et al. The effect of copper pre-cleaning on graphene synthesis. Nanotechnology, 2013, 24(36): 365602.

[19] FAGGIO G, CAPASSO A, MESSINA G, et al. High-temperature growth of graphene films on copper foils by ethanol chemical vapor deposition. The Journal of Hysical Chemistry C, 2013, 117(41): 21569-21576.

[20] LI X, ZHU Y, CAI W, et al. Transfer of large-area graphene films for high-performance transparent conductive electrodes. Nano Letters, 2009, 9(12): 4359-4363.

[21] LI X, CAI W, AN J, et al. Large-area synthesis of high-quality and uniform graphene films on copper foils. Science, 2009, 324(5932): $1312-1314$.

[22] COSTA S, TRIPISCIANO C, BOROWIAK-PALEN E, et al. Comparative study on purity evaluation of singlewall carbon nanotubes. Energy Conversion and Management, 2008, 49(9): 2490-2493.

[23] MAILRD L M, PIMENTA M A, DRESSELHAUS G, et al. Raman spectroscopy in graphene. Physics Reports, 2009, 473(5): 51-87.

[24] DRESSElHaus M S, DRESSElhaus G, JORIO A. Raman spectroscopy of carbon nanotubes in 1997 and 2007. The Journal of Physical Chemistry C, 2007, 111(48): 17887-17893.

[25] FERRARI A C, MEYER J C, SCARDACI V, et al. Raman spectrum of graphene and graphene layers. Physical Review Letters, 2006, 97(18): 187401.

[26] WANG Y Y, NI Z H, YU T, et al. Raman studies of monolayer graphene: the substrate effect. The Journal of Physical Chemistry C, 2008, 112(29): 10637-10640.

[27] NAIR R R, BLAKE P, GRIGORENKO A N, et al. Fine structure constant defines visual transparency of graphene. Science, 2008, 320(5881): 1308. 